\title{
CONTRIBUTION A LA CONNAISSANCE DE TROIS CHIRONOMIDES DE CAMARGUE ET DES MARISMAS DU GUADALQUIVIR
}

\author{
[Diptères]
}

par H. Iaville et J.-N. Tourene.

Dans une précédente note [TourenQ 1966], l'un de nous a donné un premier relevé faunistique des Chironomides adultes récoltés en Camargue du mois de juin au mois de décembre 1965. Il y avait noté la présence de l'espèce Cryptochironomus deribae décrite du Soudan par Fremman [1957]. Or, l'étude d'un paratype obligeamment prêté par ce dernier et la récente découverte aux bords de la Mer Baltique (Ostsee) d'une forme tout à fait semblable à la forme camarguaise [FITTKAU, in litt.] nous ont amené à donner un nouveau nom à la forme européenne, que nous avons également récoltée dans l'extrême sud de l'Espagne (Marismas du Guadalquivir).

Nous la rangeons dans le sous-genre Leptochironomus PAgast. Ce sous-genre, jusqu'ici monospécifique, est représenté par l'espèce L. tener KiefF. (= balticus Pag.) [Goetghebuer 1937], avec qui elle présente le plus d'affinités morphologiques, principalement au stade imaginal.

Le nom d'espèce a été choisi du fait de la grande similitude avec l'espèce africaine $C$. deribae FreE.

Nous décrivons les imagos ( $\hat{o}$ et $q$ ) et la nymphe, obtenus par élevage, de la forme camarguaise.

\section{LEPTOCHIRONOMUS PARADERIBAE n. sp.}

\section{Imago mâle.}

Longueur : $3,5-3,9 \mathrm{~mm}$.

Longueur de l'aile : 1,9-2,4 $\mathrm{mm}(\mathrm{n}=50)$.

Coloration : tête et thorax vert; bandes mésothoraciques, mésosternum, mésopleures, deux-tiers postérieurs du postnotum, yeux et scapes brun très foncé à noir; abdomen vert avec les trois premiers segments dorsalement brunâtres: styles de l'hypopyge 
et balanciers blanchâtres; hanches, partie dorsale du fémur, tibia et tarses de $\mathrm{P}_{\mathrm{I}}, \mathrm{Ta}_{5}$ de $\mathrm{P}_{\mathrm{II}}$ et $\mathrm{p}_{\mathrm{II}}$ brun.

Tête : tubercules frontaux très petits et arrondis (visibles à un grossissement de $300 \times) ; 9$ soies au vertex, 14-16 $(\mathrm{n}=4)$ au clypeus; longueurs en $\mu(n=3)$ des articles $1-4$ des palpes : 48, $124,140,206$; antennes à 12 articles, $\mathrm{AR}=2,4-3,4 \quad(\mathrm{n}=5)$, $M=2,8$; les articles $2-11$ ont un seul verticille de soies.

Thorax : les deux lobes du pronotum se joignent dans leur partie médiane le long d'une suture et s'élargissent latéralement; la zone intermédiaire est brune; la partie renflée porte 6 soies; 8-14 soies dorsomédianes $(\mathrm{dm})$ sur deux rangées, 10-12 soies dorsolatérales (dl) sur un seul rang, 2-4 soies préalaires (pa), 1 soie supraalaire ( $\mathrm{sa}$ ); scutellum avec 10-14 soies alignées dans la partie médiane; spiracle et suture anepisternale très nets.

Aile : rm non assombrie; 8 macrotriches le long de $r$; fcu distal par rapport à $\mathrm{rm}$; nervure anale dépasse largement fcu; squama avec $18-23$ soies.

Pattes : longueurs en $\mu$ des articles des pattes $(n=3)$ :

\begin{tabular}{|c|c|c|c|c|c|c|c|c|}
\hline & $\mathrm{Fe}$ & $\mathrm{Ti}$ & $\mathrm{Ta}_{1}$ & $\mathrm{Ta}_{\mathrm{s}}$ & $\mathrm{Ta}_{3}$ & $\mathrm{Ta}_{+}$ & $\mathrm{Ta}_{\mathrm{j}}$ & $\mathrm{LR}$ \\
\hline $\mathrm{P}_{1}$ & 800 & 550 & 800 & 475 & 350 & 250 & 135 & 1,45 \\
$\mathrm{P}_{11}$ & 675 & 675 & 350 & 200 & 180 & 110 & 110 & 0,51 \\
$\mathrm{P}_{11 \mathrm{I}}$ & 800 & 850 & 570 & 330 & 280 & 135 & 115 & 0,67 \\
\hline
\end{tabular}

$\mathrm{LR}_{1}=1,3-1,45(\mathrm{n}=6)$.

Extrémité distale $\mathrm{Ta}_{2}, \mathrm{Ta}_{3}, \mathrm{Ta}_{4}$ de $\mathrm{P}_{\mathrm{I}}$ et $\mathrm{Ta}_{1} \dot{\mathrm{a}} \mathrm{Ta}_{4}$ de $\mathrm{P}_{1}$, barbus : ${ }^{1} \mathrm{BR}_{\mathrm{ITa} 3}=5 ; 2$ peignes tibiaux fusionnés, aux pattes II et III, armé chacun d'un épron; pulvilles bien développées.

Hypopyge (fig. 2) : pointe anale légèrement courbée, élargic à son extrémité distale avec une expansion ventrale garnie de soies et deux soies sur sa face dorsale (fig. 4); de chaque côté de la pointe anale, deux lobes latéraux avec chacun 3-4 soies $(20 \mu)$; style long, renflé dans sa partie antérieure, rétréci et courbé vers l'intérieur à son extrémité distale et terminé par une petite dent; appendice 1 court, digitiforme, avec une soie terminale dans son prolongement et $2-4$ soies dirigées perpendiculairement vers la pointe anale.

1. $\mathrm{BR}=\frac{\text { longueur de la plus longue soie du tarse indiqué }}{\text { épaisseur du même tarse }}$ 


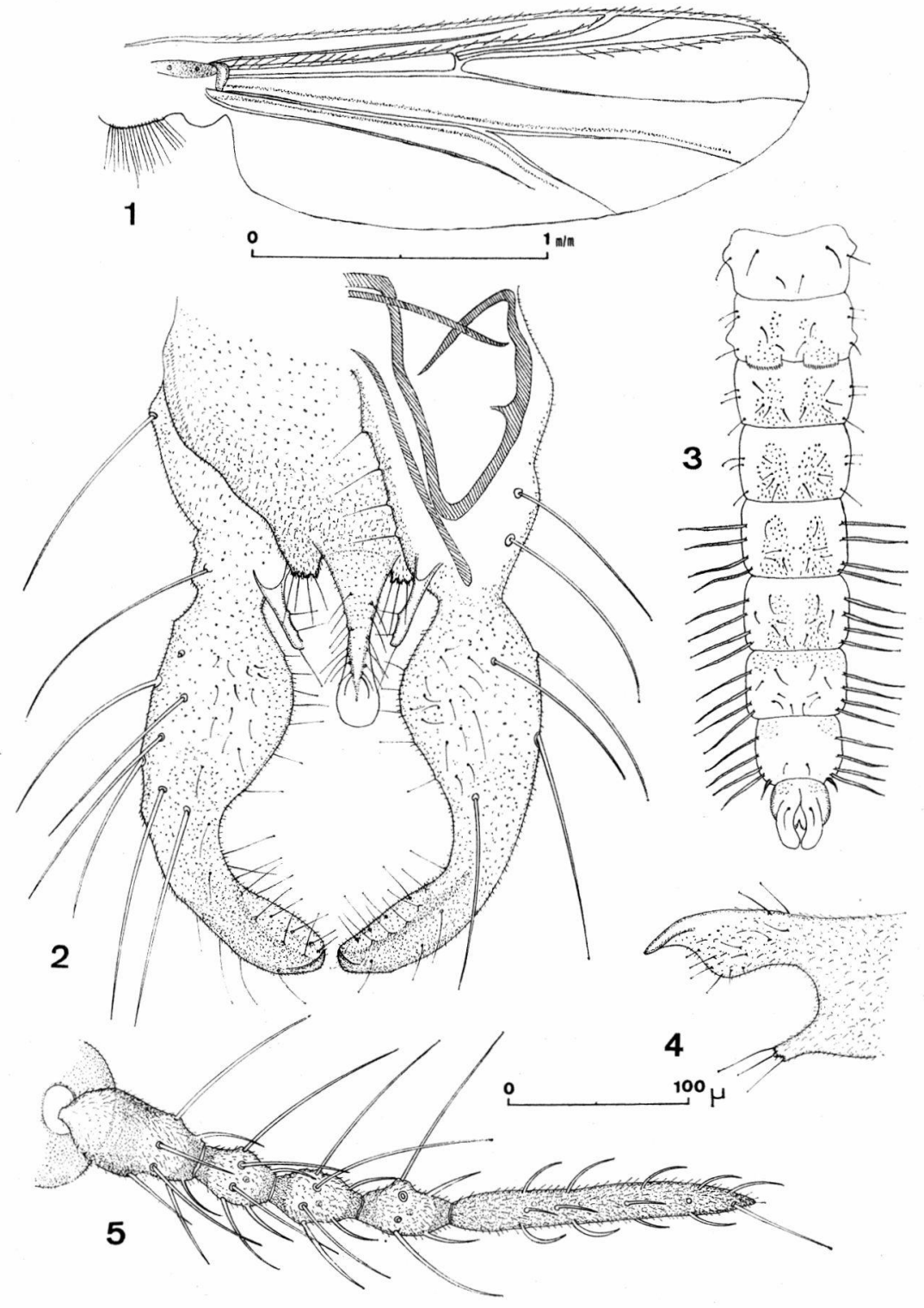

FIg. 1 à 5. - Leptochironomus paraderibae n. sp. -1 : aile $q$ : 2 : hypopyge $\sigma^{t}$ en vue dorsale; 3 : abdomen de l'exuvie nymphale montrant la chaetotaxie et l'ornementation dorsales; 4 : pointe anale de l'hypopyge $\sigma^{*}$ en vue latérale; 5 : antenne +. 
Imago femelle.

Longueur : 2,6-2,8 $\mathrm{mm}$.

Longueur de l'aile : $2,15-2,5 \mathrm{~mm}(\mathrm{n}=50)$.

Coloration : vert dans son ensemble; les parties brun froncé à noir du thorax du mâle sont ici brun clair; tibia, deux-tiers distaux de $T a_{1}, T a_{2}-T a_{5}$ de $P_{I}$ brun foncé; $T a_{2}-T a_{5}$ de $P_{I I}$ et $P_{I I I}$ brun clair.

Tête : tubercules frontaux très petits; nombre de soies du clypeus plus élevé $(18-23, n=3)$ que chez le mâle; longueurs en $\mu$ $(\mathrm{n}=3)$ des articles 1-4 des palpes : 50, 112, 140, 206; antenne (fig. 3) à 6 articles; article 2 avec à sa base un verticille de 2-3 soies, son extrémité ainsi que les segments 3,4 et 5 portent chacun un verticille de 5-6 soies longues environ de $100 \mu$ et 2 soies hyalines sensorielles, courbes et plus courtes; $6^{\mathrm{e}}$ segment avec 16-20 soies sensorielles et une seule soie subterminale droite $(28 \mu)$; longueur des segments antennaires 2-6 en $\mu(n=5)$ :

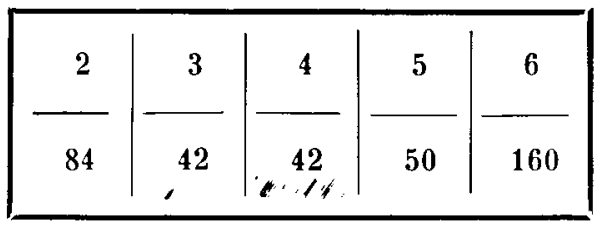

Thorax : pronotum sans partie médiane brune; chaetotaxie comparable à celle du mâle.

L'aile (fig. 1) plus large que celle du mâle, n'en diffère que par la présence du 21 macrotriches le long de $r$ et $r_{1}$ et de 13 macrotriches sur la moitié distale de $r_{4^{+5}}$.

\section{Nymphe mâ'e.}

Longueur : 4,70 $\mathrm{mm}(\mathrm{n}=2)$.

Exuvie nymphale entièrement hyaline; les lames criblées forment deux taches brunâtres ovales, marquant le départ des deux troncs principaux de la corne thoracique qui se ramifient ensuite en de nombreux filaments; petites cornes thoraciques antérieures pointuer, longues de $140 \mu$, avec une soie subterminale de $50 \mu$ environ; bords du thorax granuleux dans leur moitié antérieure.

Abdomen : expansions latérales aux bords antérieurs du segment I et aux bords postérieurs du segment II. Segment VIII avec, à son extrémité distale, un éperon jaune et courbé long de $60 \mu$.

Face dorsale : segment II bordé du côté anal par une rangée de 46-54 crochets disposés sur deux bourrelets bien séparés; tergites II-VI garnis dans leur partie médiane de fines spinules devenant un peu plus fortes au bord anal; les bords antérieurs du tergite VII 
et latéro-antérieurs des tergites VIII et IX sont très légèrement chagrinés.

Face ventrale : bande transversale de petites spinules sur le bord antérieur du sternite II; spinules plus ou moins disposées en tourbillon de chaque côté du sternite IV; sternites V-VIII chagrinés dans chaque angle antérieur.

Chaetotaxie de l'abdomen (fig. 5)².

D I-II 2, III-V 4, VI-VII 5, VIII-IX 1.

V I 2, II-IV 3, V-VII 4, VIII 1.

L I 1, II-IV 3.

LS V-VIII 4.

48-57 $(n=5)$ soies natatoires disposées sur une seule rangée garnissent chaque lobe du segment anal; fourreaux des gonopodes courbés vers l'intérieur dépasssent largement l'extrémité de la nagcoire caudale.

\section{Affinités.}

a) Avec Cryptochironomus deribae Free. ${ }^{3}$

L'étude du paratype ô de $C r$. deribae, conservé à sec, nous a cependant permis de retrouver de nombreux caractères communs aux deux espèces notamment en ce qui concerne la coloration, la présence de tarses barbus et la veine transversale non assombrie.

Toutefois des différences ont pu être observées au niveau de l'hypopyge :

- la pointe anale de Leptochironomus paraderibae est moins longue et plus large à son extrémité.

- le style, également moins long et de forme moins exagérée, a une partie médiane moins rétrécie et une partie terminale nettement moins renflée.

- les soies garnissant les parties antérieures et terminales du style sont également plus courtes (celles des parties correspondantes de $\mathrm{Cr}$. deribae nous ayant paru plus longues que sur le dessin de Freeman [1957].

- les lobes latéraux, de part et d'autre de la pointe anale, sont plus proéminents.

Nous n'avons pas pu voir si l'espèce soudanaise présentait ou non une dent à l'extrémité du style.

2. $\mathrm{D}=$ soies dorsales.

$\mathrm{V}=$ soies ventrales.

$\mathbf{L}=$ soies latérales simples.

$\mathrm{LS}=$ Laterale Schlauborsten $=$ soies natatoires latérales.

3. Nous remercions le $D^{r}$ P. Freeman d'avoir bien voulu prêter un des paratypes de cette espèce. 


\section{b) Avec Leptochironomus tener KIEFF. ${ }^{4}$}

L'hypopyge de $L$. tener présente à l'extrémité de son style une dent signalée par Pagast sur le paratype de L. balticus (= tener), mais représentée ni par lui [PAgast 1938] ni par Brundin [1947]. Nous donnons sous forme de tableau les principaux caractères permettant de différencier les deux espèces $L$. tener et $L$. paraderibae n. sp., ainsi que les variations observées dans les formes de Camargue et des Marismas.

\begin{tabular}{|c|c|c|c|}
\hline \multirow{2}{*}{ s } & \multicolumn{2}{|c|}{ L. paraderibae n. sp. } & \multirow{2}{*}{$\begin{array}{l}\text { L. tener KIEFF. } \\
(=\text { balticus PAG. }\end{array}$} \\
\hline & Camargue & Marismas & \\
\hline \multirow[t]{2}{*}{ Abdomen } & \multicolumn{2}{|c|}{ Segments I-III dorsalement brunâtre } & entièrement vert \\
\hline & $P_{1}$ en & èrement & Ti Ta, de $P_{I}$ \\
\hline \multirow[t]{2}{*}{$\begin{array}{l}\text { Pattes : } \\
\text { parties } \\
\text { brunes }\end{array}$} & $\mathrm{Ta}_{3}$ de $\mathrm{P}_{11}$ & $\begin{array}{l}\text { Extrémité } \mathbf{T} a_{1} \\
\mathbf{T a}_{11}-\mathbf{T} \mathbf{a}_{\mathbf{v}} \text { de } \mathbf{P}_{11}\end{array}$ & $\begin{array}{l}\text { Extrémité } T a_{11} \\
\text { Ta de } P_{11}\end{array}$ \\
\hline & $\mathrm{Ta}_{\mathrm{s}}$ de $\mathrm{P}_{\mathrm{uI}}$ & $\begin{array}{l}\text { Extrémité } \mathrm{Ta}_{11} \\
\mathbf{T a}_{11 \mathrm{x}}-\mathrm{T} \mathbf{a}_{\mathrm{v}} \mathrm{de} \\
\mathbf{P}_{11 \mathrm{I}}\end{array}$ & $\begin{array}{l}\text { Extrémité } \mathbf{T a}_{1} \\
\mathrm{Ta}_{11} \text { de } \mathrm{P}_{11}\end{array}$ \\
\hline Tarses & \multicolumn{2}{|c|}{ barbus } & barbus \\
\hline Longueur & $3,2-3,9 \mathrm{~mm}$ & $4,3-4,6 \mathrm{~mm}$ & $3,1 \mathrm{~mm}$ \\
\hline I. aile & $1,9-2,4 \mathrm{~mm}$ & $2,7 \mathrm{~mm}$ & $1,7 \mathrm{~mm}$ \\
\hline $\mathbf{I}_{\lrcorner} \mathbf{R}_{\mathrm{I}}$ & $1,3-1,45$ & 1,37 & $1,5-1,6$ \\
\hline AR & $2,3-3,4$ & $2,6-3$ & $1,6-1,9$ \\
\hline $\begin{array}{l}\text { Hypopyge : } \\
\text { pointe } \\
\text { anale } \\
\text { appendice } 1\end{array}$ & $\begin{array}{l}\text { courte, arrondi } \\
\text { ment. }\end{array}$ & $\begin{array}{l}\text { courbée ventrale- } \\
\text { urt }\end{array}$ & $\begin{array}{l}\text { longue, progressi- } \\
\text { vement retrécie, } \\
\text { droite. } \\
\text { long }\end{array}$ \\
\hline
\end{tabular}

4. Nous remercions également le $D^{r}$ F. Reiss de l'envoi de deux imagos $\sigma$ de $L$. tener provenant d'un étang de Andelshofen, près de Ueberlingen (Lac de Constance). 
L'exuvie nymphale de $L$. paraderibae $n$. sp. et de l'exuvie de L. balticus décrite par Pagast [1931] ont en commun les caractères suivants :

- cornes thoraciques ramifiées en de nombreux filaments;

- partie antérieure du thorax granuleuse;

- segment abdominal II avec deux bourrelets de crochets et deux expansions latéro-postérieures;

- 4 soies natatoires aux segments V-VIII;

- 1 éperon jaune de 60-65 $\mu$ de chaque côté du segment VIII;

- un nombre équivalent de soies natatoires (50 env.) sur chaque lobe anal.

L'ornementation des segments abdominaux présente toutefois des différences, comme le montre le tableau ci-après :

\begin{tabular}{|c|c|c|}
\hline Exuvie nymphale & L. paraderibae n. sp. & L. tener KIEFF. \\
\hline Longueur & $4,7 \mathrm{~mm}$ & $3,8 \mathrm{~mm}$ \\
\hline $\begin{array}{l}\text { L. petite corne } \\
\text { thoracique }\end{array}$ & $140^{\mu}$ & $90 \mu$ \\
\hline $\begin{array}{l}\text { Face dorsale : } \\
\text { Segments II, VII, VIII } \\
\text { et IX }\end{array}$ & très fines spinules & nus \\
\hline Face ventrale : & & \\
\hline Segment I & nu - expansions latérales & $\begin{array}{l}\text { spinules } \\
\text { latéro-antérieures }\end{array}$ \\
\hline Segment II & nu & $\begin{array}{l}\text { deux rangées antérieu- } \\
\text { res de spinules }\end{array}$ \\
\hline Segment V-VIII & $\begin{array}{l}\text { spinules } \\
\text { latéro-antérieures }\end{array}$ & nus \\
\hline
\end{tabular}

\section{Ecologie.}

Du point de vue écologique, $L$. paraderibae n. sp. peut être considérée, du moins en Camargue, comme une espèce halobie, puisque des larves, prélevées fin juillet, avant l'assèchement du marais de la Baisse Salée, se trouvaient dans une eau dont la salinité de l'ordre de $42 \mathrm{~g} / \mathrm{l}$ est supérieure à celle de la Méditerranée; c'est la seule station où on la rencontre. Aux Marismas, les récoltes en vol ne nous permettent pas d'affirmer si les larves 
colonisent uniquement les biotopes saumâtres. Mais nous retenons également sa découverte au bord de la Mer Baltique.

\section{Dates des captures.}

Des larves prélevées dans le marais de la Baisse Salée et mises en élevage le 5 juin 1965 se sont métamorphosées du 15-VI-65 au 6-VII-65. Des prélèvements effectués au même endroit le 25 juillet 1965 ont libéré des imagos $\hat{o}$ et $q$ du 9-VIII-65 au 26VIII-65. De nombreux adultes ont également été récoltés en vol toujours aux environs du marais de la Baisse Salée, du 15 au 20 juin 1965. Les adultes des Marismas ont été capturés pendant les mois de janvier et de février 1966, mais signalons que les chasses n'y ont été effectuées que pendant les quatre premiers mois de l'année 1966.

L'holotype, l'allotype et plusieurs paratypes de Leptochironomus paraderibae $\mathrm{n}$. sp. se trouvent dans la collection LAviLLE (Laboratoire de Zoologie, Toulouse); des paratypes sont également déposés dans la collection FrtrkaU (Plön).

\section{POLYPEDILUM PHARAO KIEFFER, 1924.}

Deux imagos de cette espèce, décrite par Kiefrer sur 5 exemplaires $(4 \stackrel{+}{4}, 1 \quad \hat{b})$ trouvés au nord de l'Egypte, ont été signalés plus récemment de Bulgarie près de Varna, à l'embouchure de la Provadiya par Strenzke [1951], qui donne un nouveau dessin de l'hypopyge.

L'adulte $\delta$ présente les caractères du genre Polypedilum définis par Edwards [1929]. Il s'en différencie cependant par la présence de tubercules frontaux, par un style court et trapu, ainsi que par l'absence d'éperon au tibia antérieur.

L'exuvie nymphale (fig. 9), obtenue par élevage, présente une corne thoracique ramifiée en de nombreux et fins filaments comme on en rencontre seulement dans le genre Stictochironomus du groupe des "Chironomariae connectentes 》[LENz 1941].

Le peigne anal est armé d'un plus grand nombre de puissantes épines, dont 2-5 sont dirigées plus ou moins latéralement (fig. 8). L'intersegment $5 / 6$ est également garni de petites épines. Les sternites II, III, IV sont légèrement chagrinés avec en plus aux bords latéraux du sternite IV de petites spinules disposées en «tourbillon ».

L'exuvie paraît présenter suffisamment de caractères originaux pour qu'on la place dans un groupe voisin des groupes laetum, nebulosum et convictum du genre Polypedilum dont le nombre de filaments, d'ailleurs plus larges, de la corne thoracique ne 
dépasse pas 8 . Mais seule la connaissance de la larve nous permettra de situer cette espèce de façon plus précise du point de vue systématique.

\section{Imago mâle.}

Longueur : 4,6-5,2 $\mathrm{mm}$.

Longueur de l'aile : $2,42-2,78 \mathrm{~mm}(\mathrm{n}=6)$.

Coloration : bandes mésothoraciques, postnotum, mésosternum, scapes et hanches brun foncé. Pronotum, scutellum et abdomen brun clair. Pattes, balanciers, soies du panache antennaire jaune à brun très clair.

Tête : présence de tubercules frontaux; 15 soies $(90 \mu)$ au vertex, 16-19 soies $(130 \mu)$ au clypeus; longueurs (en $\mu, \mathrm{n}=2$ ) des articles 1-4 des palpes : 50, 130, 125, 200; antennes à 14 articles, $A R=2,7-3,3$; articles 2-5 à un seul verticille de soies, articles 6-13 à deux verticilles, article terminal à $9-10$ soies sensorielles.

Thorax : pronotum réduit, dépourvu de soies, interrompu dans sa partie médiane; mésonotum : 17-19 soies $\mathrm{dm}$ sur deux rangées, 24-31 soies dl sur trois, deux ou une rangées. 0 soie sa, 6-8 soies pa; scutellum à $20-28$ soies.

Aile : 9 taches réparties comme suit : 3 dans $R, 3$ dans $M$, la plus importante le long de $\mathrm{Cu}_{2}, 2$ dans $\mathrm{An}$; squama à 15 soies; une rangée de macrotriches tout le long de $r$ et $r_{1}$ et sur la moitié distale de $r_{4^{+}}$; ces deux nervures légèrement assombries après $\mathrm{rm}$; fcu légèrement distal par rapport à $\mathrm{rm}$.

Pattes : tibia antérieur sans éperon; deux peignes tibiaux séparés à la patte moyenne, seul l'extérieur porte un éperon; ceux des tibias postérieurs sont contigus, avec un éperon également au peigne extérieur; pulvilles bien développées; soies courtes en forme de crochet sur le cinquième distal du métatarse de $P_{I I}$; longueurs en $\mu$ des articles des pattes $(\mathbf{n}=3)$ :

\begin{tabular}{|c|c|c|c|c|c|c|c|c|}
\hline & $\mathrm{Fe}$ & $\mathrm{Ti}$ & $\mathrm{Ta}_{1}$ & $\mathrm{Ta}_{2}$ & $\mathrm{Ta}_{3}$ & $\mathrm{Ta}_{4}$ & $\mathrm{Ta}_{5}$ & L.R \\
\hline$P_{1}$ & 887 & 725 & 1035 & 575 & 500 & 372 & 180 & 1,42 \\
\hline $\mathbf{P}_{11}$ & 1035 & 825 & 550 & 312 & 250 & 185 & 125 & 0,66 \\
\hline$P_{111}$ & 1035 & 1025 & 737 & 412 & 375 & 225 & 137 & 0,77 \\
\hline
\end{tabular}

$\mathrm{BR}_{\mathrm{Tn} 3}=5,5-6$.

Hypopyge : pointe anale longue, à bords parallèles (en vue dorsale) et effilée à son extrémité (en vue latérale); appendice 1a long, aminci à son extrémité distale et courbé vers la face 
ventrale (en vue latérale); appendice 2 à bords parallèles et à terminaison plus ou moins anguleuse, garni dorsalement de $\mathbf{1 6}$ à 20 soies courbées vers l'avant et d'une soie plus longue $(80 \mu)$ droite dirigée à $45^{\circ}$ par rapport au plan de symétrie de l'hypopyge; style court, trapu, garni sur la face interne de soies longues de $40 \mu$; les soies du côté externe atteignent $150 \mu$.

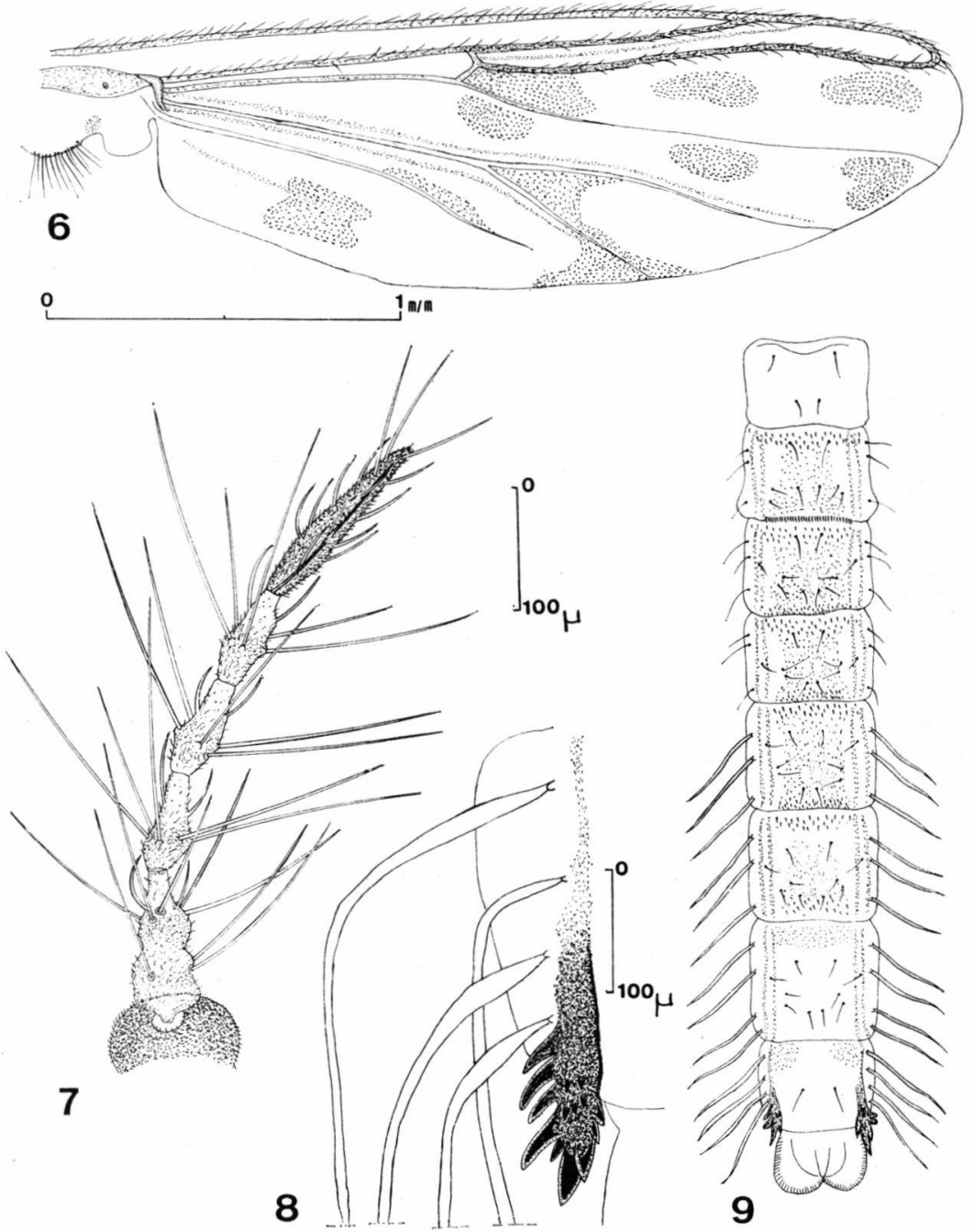

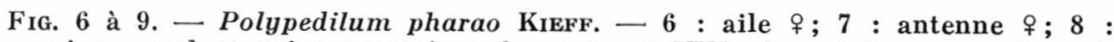
peigne anal et soies natatoires du segment VIII; 9 : abdomen de l'exuvie nymphale montrant la chaetotaxie et l'ornamentation dorsales. 
Imago femelle.

Longueur : 3,4-3,8 $\mathrm{mm}$.

Longueur de l'aile : $2,2-3 \mathrm{~mm}(\mathrm{n}=5)$.

Coloration semblable à celle dı mâle.

Tête : soies du clypeus plus nombreuses (20-30) que chez le mâle.

Antenne (fig. 7) à 6 articles; articles 1 et 6 brun foncé; article 2 à 2 verticilles de 4 et 7 soies $(200 \mu)$; articles 3 à 5 à 1 seul verticille de 6 soies; articles 2 à 5 avec chacun 2 soies sensorielles courbes subdistales; article 6 à $3-4$ verticilles de soies sensorielles hyalines, courbes, et 3 soies droites subterminales $(110 \mu)$; longueurs des articles antennaires $2-6$ en $\mu(n=4): 136,78,75,78,159$.

Chaetotaxie de la tête et du thorax comme chez le mâle avec toutefois un plus grand nombre de soies dm (37-44).

Aile (fig. 6) semblable à celle du mâle avec des macrotriches tout le long de $\mathbf{r}_{4^{+}}$.

Pattes : présence de soies courtes en forme de crochet sur les $2 / 3$ distaux du métatarse de $P_{I I}$ et sur le $1 / 6$ terminal du métatarse de $P_{1 \cdot \text {. }}$.

Longueurs en $\mu$ des articles des pattes $(n=10)$ :

\begin{tabular}{|c|c|c|c|c|c|c|c|c|}
\hline & $\mathrm{Fe}$ & $\mathrm{Ti}$ & $\mathrm{Ta}_{1}$ & $\mathrm{Ta}_{2}$ & $\mathrm{Ta}_{3}$ & $\mathrm{Ta}_{\mathrm{a}_{*}}$ & $\mathrm{Ta}_{\mathrm{sis}}$ & LR \\
\hline$P_{1}$ & 930 & 700 & 970 & 450 & 450 & 350 & 200 & 1,38 \\
\hline$P_{11}$ & 1100 & 960 & 600 & 300 & 250 & 190 & 110 & 0,62 \\
\hline $\mathbf{P}_{111}$ & 1200 & 1200 & 800 & 408 & 390 & 210 & 120 & 0,66 \\
\hline
\end{tabular}

\section{Nymphe.}

Longueur : 5,3-6,7 $\mathrm{mm}$.

Exuvie hyaline; suture antérieure du thorax, une zone plus ou moins annulaire au départ des fourreaux alaires, bords latéraux du $8^{\text {e }}$ tergite abdominal légèrement brun; peigne anal brun plus foncé.

Corne thoracique ramifiée en de fins et nombreux filaments avec trois troncs principaux débouchant sur une lame criblée ovoïde de $100 \mu$ environ; petites cornes thoraciques $(80 \mu)$ obtuses, avec une courte soie $(30 \mu)$ subterminale; bords de la suture thoracique très légèrement granuleux dans leur partie médiane.

Abdomen : segment II avec 2 pseudopodes latéraux et une rangée de 70 à 90 crochets alignés sur le bord anal; peigne anal avec 2-5 fortes épines latérales et 1 ou 2 apicales; quelques épines 
plus petites garnissent sa face dorsale (fig. 8). Segments II à VI avec une bande transversale orale de petites spinules et une bande semblable de spinules dans la partie anale des segments III à VI; entre les deux, partie intermédiaire légèrement chagrinée laissant des zones arrondies glabres; intersegments $3 / 4,4 / 5$ et $5 / 6$ garnis de spinules; bord oral du segment VII et angles antérieurs du segment VIII garnis de très fines spinules; sternites II, III et IV, légèrement chagrinés; sternite IV avec en plus une zone de spinules disposées en tourbillon sur les bords latéro-postérieurs.

Chaetotaxie de l'abdomen (fig. 9) :

D I 2, II-VII 5, VIII 1.

V II-IV 2, V-VII 4, VIII 1.

L II-IV 3.

\section{LS V-VI 3, VII-VIII 4.}

50-60 soies natatoires disposées sur une seule rangée garnissent chaque lobe du segment anal.

\section{Ecologie.}

C'est, en Camargue, une des espèces les plus abondantes. Ses larves colonisent aussi bien les milieux d'eau douce (Canal du Rendez-vous) que les milieux d'eau saumâtre; ces milieux ont été précédemment définis [Touneng 1966]. On peut donc la considérer comme une espèce euryhaline.

\section{Dates des captures.}

En vol, sur le territoire de la Tour du Valat : 15-VI-65: 10 , 1 ₹; 21-VI-65 : 5 ô, 10 q; 28-VI-65 : 8 ô; 29-VI-65 : 30 ô, 10 \%;

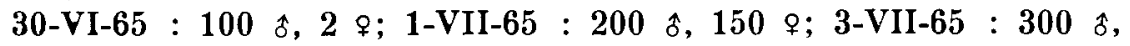
150 \%; 10-VII-65 et 27-VII-65 : vols très denses d'imagos $\hat{o}$ et $q$;

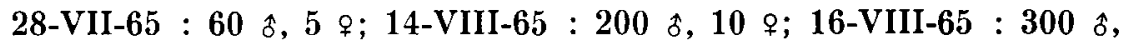

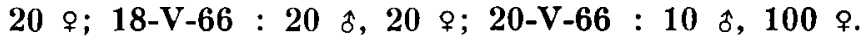

Des pontes ont été recueillies dans des milieux différents et mises en élevage au laboratoire de la Tour du Valat. Elles nous ont permis d'obtenir des imagos $\hat{o}$ et $q$ entre le $22^{\circ}$ et le $41^{\circ}$ jour suivant, ce qui correspond à un cycle de développement rapide. Les éclosions se sont succédées de fin mai à début septembre, avec un maximum d'adultes durant les mois de juin et juillet.

Nous avons également récolté de nombreux imagos $\delta$ et $q$ en vol, au sud de l'Espagne, dans la zone marécageuse des Marismas à l'embouchure du Guadalquivir en janvier et février 1966. Les formes espagnoles sont tout à fait semblables aux formes de Camargue, avec toutefois une pigmentation plus marquée, ainsi que des taches alaires plus nettes. 
Le sous-genre Calochironomus créé par KIEFFER [1911] et caractérisé par la présence de taches sur les ailes des imagos a été mis en synonymie avec le sous-genre Chironomus s. tr. par BRundiN dans un récent travail "Afrikanische Chironomiden 》(FitTkaU, in litt.).

La connaissance de l'exuvie nymphale et de l'imago $t$, non décrits, tout à fait conformes au sous-genre Chironomus s. str., nous permet d'y ranger l'espèce hexastictus, le seul caractère vraiment original de l'adulte restant la présence de six taches alaires (fig. 10), l'exuvie, elle, se distinguant par sa petite taille $(6,2-$ $6,6 \mathrm{~mm})$ et par le nombre réduit (2-3) d'épines constituant l'éperon anal.

\section{Imago mâle.}

Longueur : 4,5-5,3 mm $(\mathrm{n}=10)$.

Longueur de l'aile : $2,6-3,1 \mathrm{~mm}(\mathrm{n}=50)$.

Coloration : tête, thorax, abdomen verdâtre sur le vivant (jaunâtre dans l'alcool); bandes mésonotales, postnotum, mésosternum, antennes brun roux; yeux brun foncé; balanciers, palpes labiaux blanchâtres; pattes également blanchâtre avec une tache brune à l'extrémité subterminale des trois fémurs; extrémité distale des tarses $1-4$ bruns, le $5^{\circ}$ (parfois le $4^{e}$ également) brun entièrement.

Tête : tubercules frontaux tubulaires $(54 \mu)$; à l'extrémité de chaque branche du $\mathrm{V}$ du vertex, petite corne pointue d'un brun très foncé à noir (fig. 15) ; 19 -35 soies sur deux rangées au vertex, 18 - 31 soies au clypeus; longueurs en $\mu$ des articles 1 - 4 des palpes labiaux $(n=3): 44,180,190,225$; antennes à 12 articles : article 2 avec un seul verticille de soies sensorielles courtes $(60 \mu)$, articles 2 - 5 avec en plus un verticille de longues soies, articles $6-11$ avec seulement un verticille de longues soies; $A R=2,8-3,3(n=8)$.

Thorax : pronotum formant un collier continu avec 7 soies latérales; mésonotum avec 11 - 15 soies dorsomédianes sur deux rangées, 15 - 18 soies dorsolatérales irrégulièrement réparties, 4 - 5 soies préalaires, 1 soie supraalaires; scutellum avec $12-16$ soies plus ou moins disposées sur deux rangées.

Aile (fig. 11) : nervure transversale $\mathrm{rm}$ brune; 6 taches irisées réparties comme suit : 2 dans $R, 1$ dans $M, 1$ dans $C u$ le long de $\mathrm{Cu}_{1}$ et $\mathrm{cu}_{2}, 1$ le long de la nervure anale, 1 allongée et plus nette également dans la zone anale An; 38-45 macrotriches le long de r, 25-29 le long de $r_{1}, 27-41$ dans la moitié distale de $r_{4^{+} 5}$; fcu sous $\mathrm{rm}$, nervure anale dépasse largement $\mathrm{fcu}$; lobe anal à angle aigu; squama avec $12-15$ soies. 
Pattes : longueurs (en $\mu$ ) des articles des pattes $(\mathrm{n}=3)$.

\begin{tabular}{|l|r|r|r|r|r|r|r|r|}
\hline & $\mathrm{Fe}$ & $\mathrm{Ti}$ & $\mathrm{Ta}_{1}$ & $\mathrm{Ta}_{2}$ & $\mathrm{Ta}_{3}$ & $\mathrm{Ta}_{4}$ & $\mathrm{Ta}_{5}$ & $\mathrm{LR}$ \\
\cline { 2 - 6 } $\mathrm{P}_{1}$ & 1108 & 929 & 1667 & 879 & 620 & 508 & 268 & $\mathbf{1 , 7 9}$ \\
$\mathrm{P}_{11}$ & 1187 & 1092 & 646 & 381 & 276 & 196 & 138 & 0,59 \\
$\mathrm{P}_{111}$ & 1362 & 1383 & 1020 & 608 & 479 & 279 & 175 & 0,66 \\
\hline
\end{tabular}

Écaille antérieure des tibias obtuse; 2 peignes tibiaux aux pattes II et III occupant les $3 / 4$ du pourtour du tibia, chacun armé d'un éperon; les pulvilles atteignent la moitié de la longueur des griffes;

$\mathrm{Br} \mathrm{Ta}_{2}=3,7-4,4$; soies ventrales en forme de crochet sur le $1 / 5$ distal des métatarses de $P_{\text {II }}$ et $P_{\text {III. }}$.

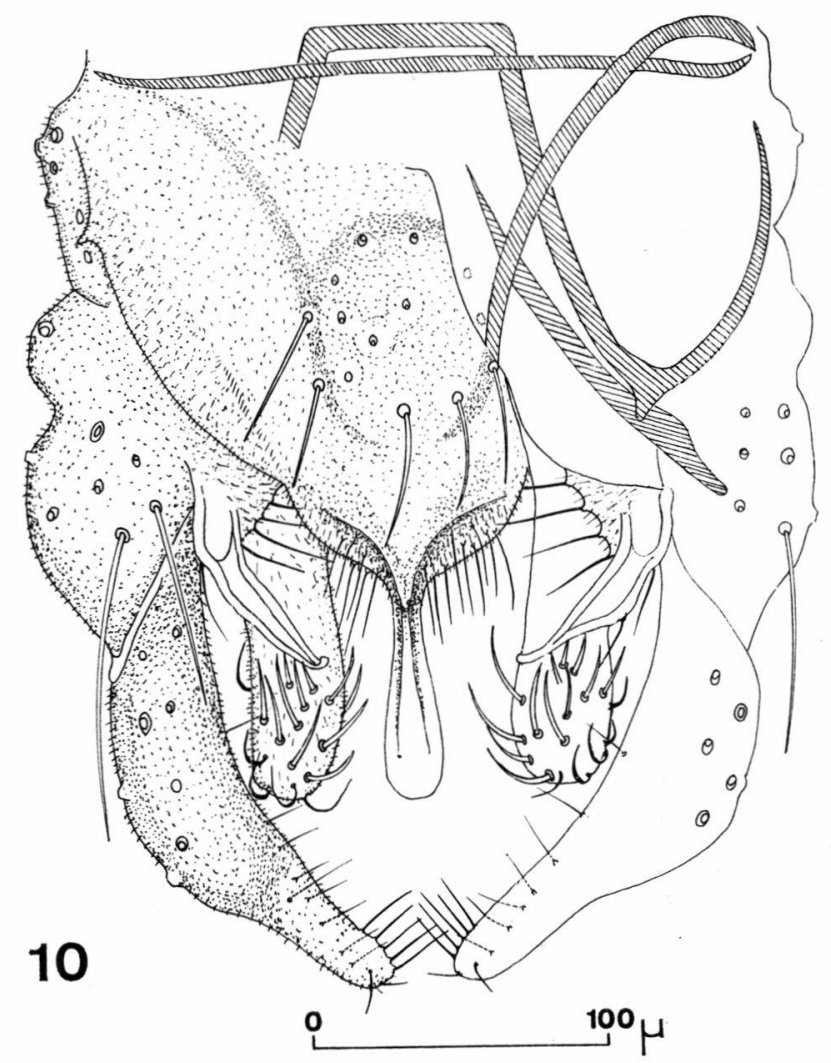

Fig. 10. - Chironomus hexastictus KiefF. - Hypopyge $\sigma^{\circ}$ en vue dorsale. 
Hypopyge (fig. 10) : pour sa description, nous avons adopté la nomenclature utilisée par STRENzKE [1959]; tergite anal avec 13 - 17 soies médianes de $80 \mu$ de long; pointe anale longue, distalement élargie et courbée vers la face ventrale, avec 8-10 soies $40-55 \mu)$ de part et d'autres de sa base; appendice 1a formant un lobe, avec 8 - 6 soies dirigées vers l'intérieur ou vers l'extrémité; le claspette, suivant l'orientation de la préparation, se présente différemment (fig. 16) : le plus souvent renflé dans sa partie médiane, pointu et courbe à son extrémité; les paramères, droits, à bords parallèles, garnis distalement de 12-15 soies courbées vers l'avant et de quelques soies courtes sur la face externe, atteignent la moitié de la longueur du style; ce dernier, renflé et légèrement brun à sa base, blanc et étroit à son extrémité, présente 5-6 soies internes $(35 \mu), 1$ soie dorsale, et $7-9$ soies ventrales.

\section{Imago femelle.}

Longueur : 4,8-5,8 $\mathrm{mm}(\mathrm{n}=5)$.

Longueur de l'aile : $2,6-3,1 \mathrm{~mm}(\mathrm{n}=10)$.

Coloration : semblable à celle du mâle.

Tête : longueur (en $\mu$ ) des articles 1 - 4 des palpes labiaux : 48, $152,188,260$; antennes à 6 segments; segments I et VI, renflements des segments II à V plus brun que le reste de l'antenne; soies du verticille basal du segment II longues de $120-150 \mu$; soies des verticilles I - V longues de $210-275 \mu ; 2$ soies sensorielles courbes $(70 \mu)$ insérées à l'endroit où les segments se rétrécissent en goulot; segment VI avec une soie subterminale de $105 \mu$ environ; longueur en $\mu$ des articles I - VI : 168, 128, 128, 124, $180(\mathrm{n}=3)$.

Aile plus large que celle du mâle; une rangée de soies sur $\mathrm{r}_{1}$; $r_{4^{+} 5}$ avec des soies sur un rang au $1 / 3$ proximal et sur deux rangs aux $2 / 3$ distaux; lobe anal à angle obtu; squama à 22 soies.

Pattes : longueur (en $\mu$ ) des articles des pattes $(n=3)$.

\begin{tabular}{|c|c|c|c|c|c|c|c|c|}
\hline & $\mathrm{Fe}$ & $\mathrm{Ti}$ & $\mathrm{Ta}_{1}$ & $\mathrm{Ta}_{2}$ & $\mathrm{Ta}_{3}$ & $\mathrm{Ta}_{4}$ & $\mathbf{T a}_{5}$ & LR \\
\hline$P_{1}$ & 1300 & 987 & 1725 & 850 & 675 & 600 & 262 & 1,74 \\
\hline$P_{11}$ & 1250 & 1150 & 662 & 350 & 275 & 187 & 150 & 0,57 \\
\hline$P_{111}$ & 1400 & 1500 & 1062 & 587 & 475 & 275 & 187 & 0,70 \\
\hline
\end{tabular}

$\mathrm{LR}_{\mathrm{I}}=1,7-1,9(\mathrm{n}=5)$; presque 1,5 sur l'exemplaire décrit par KIEFFER [1924].

Soies ventrales en forme de crochet sur les $4 / 5^{\circ}$ distaux de $P_{\text {Ir }}$ et $\mathbf{P}_{\mathrm{III}}$. 


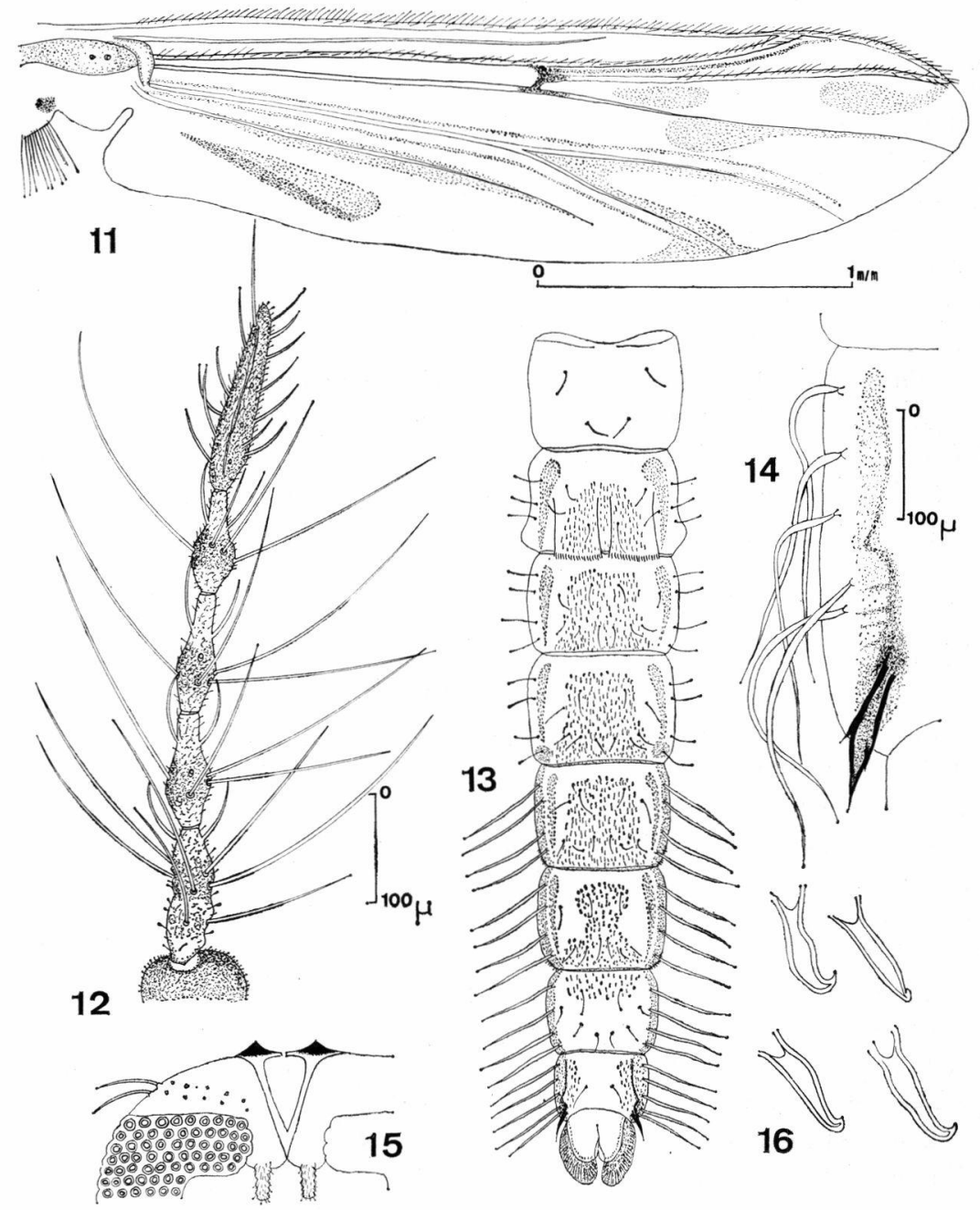

FIG. 11 à 16. - Chironomus hexastictus KIfFF. - 11 : aile $\delta^{\star} ; 12$ : antenne $q$; 13 : abdomen de l'exuvie nymphale montrant la chaetotaxie et l'ornementation dorsales; 14 : éperon anal et soies natatoires du segment VIII; 15 : vertex et tubercules frontaux en vue dorsale; 16 : hypopyge $\sigma^{\circ}$ : variation du claspette.

\section{Nymphe.}

Longueur : 6,2 - 6,6 $\mathrm{mm}$.

Exuvie nymphale hyaline, brune aux parties suivantes : zone thoracique dorsale, moitié proximale des fourreaux des pattes, base des fourreaux antennaires, bord des fourreaux alaires, bords latéraux des segments V-IX, bande de chaque côté des tergites 
II-VI; éperon anal brun foncé, formé d'une seule grande épine principale et de 1 ou 2 petites épines latérales (fig. 14) du type c et d, représentés par ANDERSÉN [1949].

Corne thoracique très ramifiéc avec deux taches brunâtres ovoïdes marquant l'emplacement de la lame criblée sur le thorax; petites cornes thoraciques pointues longues de $75 \mu$ avec une soie subterminale de $45 \mu$.

Thorax granuleux dans sa moitié dorsoantérieure.

Abdomen : segment II avec deux expansions latérales; une rangée de crochets placés sur deux bourrelets presque contigus occupant la moitié du bord anal du tergite; segment VIII avec deux éperons latéraux $(130 \mu)$ s'effilant distalement; tergites II-VI très légèrement chagrinés dans leur partie médiane; spinule très fines à la partie supérieure du tergite VII et aux parties latérales du tergite VIII; deux tourbillons de spinules aux bords latéro-postérieurs du segment IV. Très fines spinules dans les angles postérieurs des sternites $V$ et VII et sur les membranes des intersegments $4 / 5,5 / 6$ et $6 / 7$.

Charetotaxie de l'abdomen (fig. 13) :

D I 2, II 3, III-VI 5, VII 4, VIII 1.

V I 2, II-III 3, IV-VI 4, VII 3, VIII 1 .

L I 0, II 3, III-IV 4 .

LS V-VII 4, VIII 5.

67 soies $(n=1)$ alignées sur deux ou trois rangées à chaque lobe du segment anal.

\section{Écologie.}

Les larves de cette espèce ont été recueillies dans les mêmes milicux d'eau douce (Rizières, Canal du Rendez-vous) et saumâtre (Marais du Saint-Seren et de la Baisse-Salée) où vit également Polypedilum pharao. Aussi peut-on la considérer comme une espèce euryhaline.

\section{Dates des captures.}

En vol, sur le territoire de la Tour-du-Valat :

27.VII.65 : 17 s̊ (Rizières) ; 27.VII.65 : 3 \& (Canal du Rendez-vous). 28.VII.65 : 70 $\hat{\circ}$ (Rizières); 20.XI.65 : 100 of (Canal du Rendez-vous).

De nombreux imagos $\delta$ et $q$ de cette espèce ont également été capturés en vol pendant les mois de février, mars et avril aux Marismas (Espagne), région marécageuse où se retrouvent des biotopes comparables à ceux de Camargue.

Des pontes recueillies dans ces différents milieux et mises en élevage au laboratoire ont donné des imagos of et $q$ du $42^{\mathrm{e}}$ 
au $80^{\circ}$ jour suivant. En Camargue, deux périodes d'éclosion ont été observées soit dans la nature soit dans nos élevages : une fin juilletdébut août, l'autre en novembre.

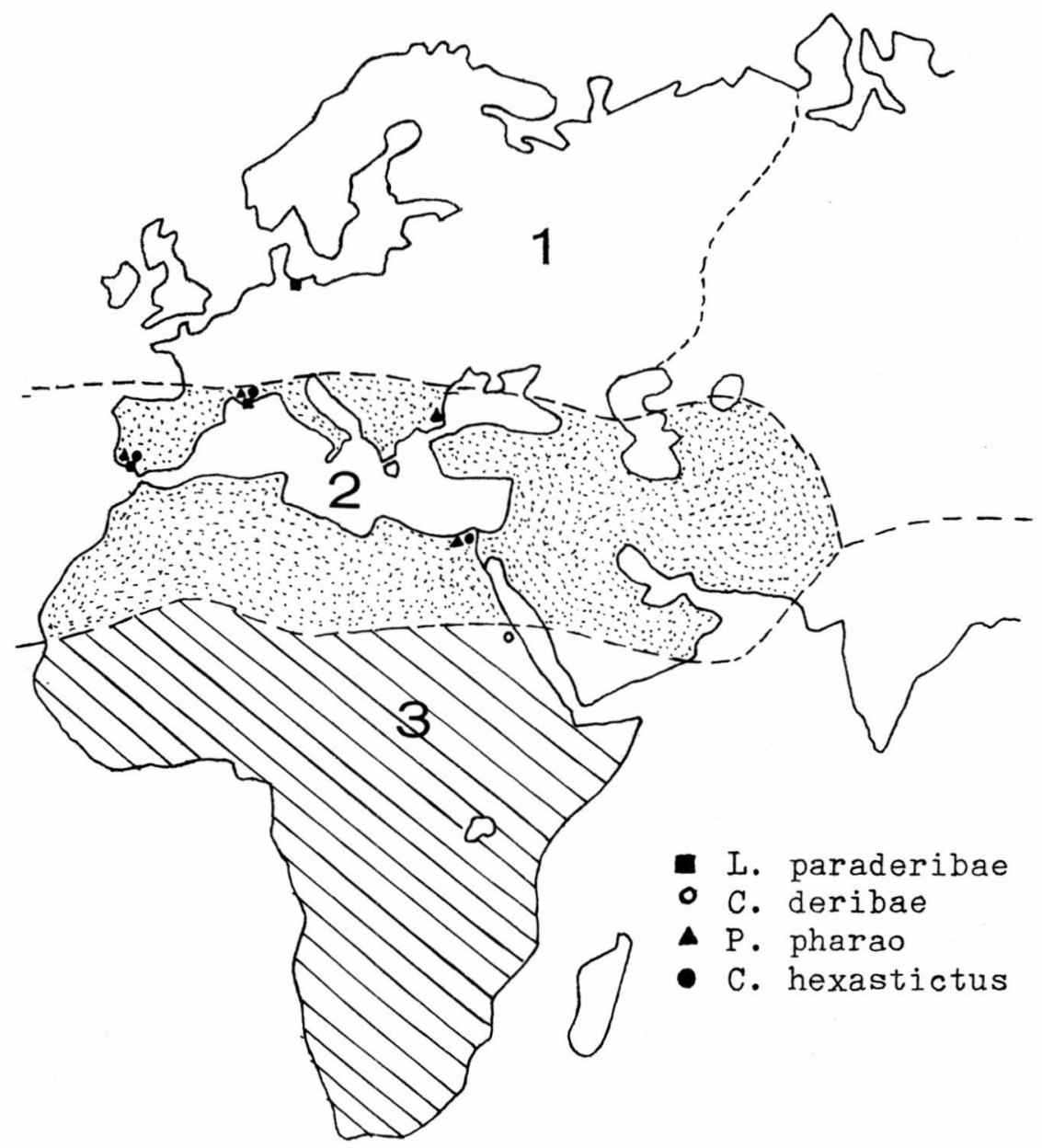

FIG. 17. - Carte [d'après SÉGUY 1950] de la répartition géographique actuellement connue des espèces étudiées.

1 : province européenne.

2 : sous-région méditerranéenne.

3 : région éthiopienne. 


\section{CONCIUSION}

Nous avons rassemblé dans cette note l'étude systématique de trois espèces de la tribu des Chironomini. Deux d'entre elles, Polypedilum pharao KIEFF. et Chironomus hexastictus KIEFF, se répartissent dans la zone méditerranéenne et, compte tenu des stations de récolte en Camargue, peuvent être considérées comme euryhalines; la troisième, Leptochironomus paraderibae $\mathrm{n}$. sp., très voisine d'une espèce soudanaise, a été trouvée soit en milieu saumâtre (Camargue), soit près des côtes de la Mer Baltique et peut, de ce fait, être tenue pour halobie.

Nous indiquons sur la figure 17 la répartition géographique actuelle de ces différentes espèces.

En Camargue, les récoltes et les élevages effectués à partir de pontes ou de larves nous ont de plus permis de donner pour ces trois Chironomides les périodes d'éclosion, ainsi que les longueurs du cycle de développement observées chez $P$. pharao et Ch. hexastictus.

\section{RÉSUMÉ}

Les stades imaginal ( $\hat{b}$ et o) et nymphal d'une nouvelle espèce Leptochironomus paraderibae sont décrits ainsi que les formes camarguaises de deux espèces à répartition méditerranéenne, Polypedilum pharao KiEfF. et Chironomus hexastictus KIEFF. Pour chacune de ces trois espèces sont également données des remarques d'ordre biologique, écologique et biogéographique.

\section{CONTRIBUTION TO THE KNOWLEDGE OF THREE CHIRONOMIDS OF THE CAMARGUE AND THE MARISMAS OF THE GUADALQUIVIR}

The imaginal and nymphal instars of the new species Leptochironomus paraderibae are described, as well as the camarguian forms of two mediterranean species, Polypedilum pharoo KIFFF. and Chironomus hexastictas KIEFF. For each of these three species, hiological and biogeographical data are also given.

\section{ZUR KENNTNIS VON DREI CHIRONOMIDENARTEN DER CAMARGUE UND DER MARISMAS DES GUADALQUIVIR}

Es werden die imaginalen und nymphalen Stadien der neuen Art Leptochironomus paraderibae wie auch die Camargueformen der beiden am Mittelmeer verbreiteten Arten (Polypedilum pharao KIEFf. und Chironomus hexastictus KIEFF.) beschrieben. Zu jeder dieser drei Arten werden ebenfalls biologische, ökologische und biogeographische Bemerkungen gemacht. 


\section{TRAVAUX CITES}

Andersen (F. S.). 1949. - On the subgenus Chironomus. Studies on the systematics and biology of Chironomidae III. Vidensk. Meddr Dansk Naturh. Foren., 3 : 1-66.

Brundin (L.). 1947. - Zur Kenntnis der schwedischen Chironomiden. Ark. Zool. Stockholm, 39 A (3) : 1-95, 25 pl.

Edwards (F. W.). 1929. - British non biting midges (Dipt. Chiron.). Trans. R. Ent. Soc. London, 77 (2) : 279-430.

Freeman (P.). 1957. - A study of the Chironomidae of Africa. Part III. Bull. Brit. Mus., 5 (9) : 1-396.

Goetghebuer (M.). 1937 a. - Tendipedidae (Chironomidae). Subfamilie Tendipedinae (Chironominae). A. Die imagines, in Lindner, die Fliegen der paläarktischen Region, 13 c : 1-128.

Kieffer (J. J.). 1911. - Description de nouveaux Chironomides de l'Indian Museum de Calcutta. Rec. Indian Mus., 6 (3) : 113-177, 2 pl.

KiefFer (J. J.). 1924-1925. - Chironomides d'Egypte. Bull. Soc. Ent. Egypte, 2-4 : 244-313.

Lenz (F.). 1941. - Die Jugenstadien der Sectio Chironomariae (Tendipedini) connectentes (s. f.; Chironominae - Tendipedinae). Arch. Hydrobiol., 38 : 1-69.

Pagast (F.). 1931. - Chironomiden aus der Bodenfauna des Usma-Sees in Kurlandl. Folia zool. Hydrobiol., 3 : 199-248.

SÉGuy (E.). 1950. - Biologie des Diptères. in Encyclopédie entomologique, XXVI : 609 p. Paris.

Strenske (K.). 1951. - Chironomiden von der bulgarischen Küste des schwarzen Meeres. Arch. Hydrobiol., Suppl. 18 (4) : 677-691.

Strenzke (K.). 1959. - Revision der gattung Chironomus. Die Imagines von 15 norddeutschen Arten und Unterarten. Arch. Hydrobiol., 56 : $1-42$,

Tourene (J.-N.). 1966. - Introduction à l'étude écologique des Chironomides de Camargue. Annls Limnol., 2, 2 : 459-465.

Laboratoire de Zoologie

de la Faculté des Sciences de Toulouse et Station biologique de la Tour du Valat, par Le Sambuc (B.-du-Rh.). 